\title{
Compressible mixing layer growth rate and turbulence characteristics
}

\author{
By A. W. VREMAN ${ }^{1}$, N. D. SANDHAM ${ }^{2}$ AND K. H. LUO \\ ${ }^{1}$ Department of Applied Mathematics, University of Twente, \\ PO Box 217, 7500 AE Enschede, The Netherlands \\ ${ }^{2}$ Department of Aeronautical Engineering, Queen Mary and Westfield College, \\ Mile End Road, London E1 4NS, UK
}

(Received 7 June 1995 and in revised form 11 March 1996)

Direct numerical simulation databases have been used to study the effect of compressibility on mixing layers. The simulations cover convective Mach numbers from 0.2 to 1.2 and all contain a fully resolved turbulent energy cascade to small spatial scales. Statistical information is extracted from the databases to determine reasons for the reduced growth rate that is observed as the convective Mach number is increased. It is found that the dilatational contribution to dissipation is negligible even when eddy shocklets are observed in the flow. Also pressure-dilatation is not found to be significant. Using an accurate relation between the momentum thickness growth rate and the production of turbulence kinetic energy together with integrated equations for the Reynolds stress tensor it is shown that reduced pressure fluctuations are responsible for the changes in growth rate via the pressure-strain term. A deterministic model for the required pressure fluctuations is given based on the structure of variable-density vortices and the assumption that the limiting eddies are sonic. Simple anisotropy considerations are used to close the averaged equations. Good agreement with turbulence statistics obtained from the simulations is found.

\section{Introduction}

An early experimental observation of the effect of Mach number on turbulence was the reduction in growth rate of the plane mixing layer (Birch \& Eggars 1973). It is now established that compressibility rather than density effects are responsible for the growth rate reduction (Brown \& Roshko 1974; Bradshaw 1977; Papamoschou \& Roshko 1988). In recent years there have been several experimental, numerical and theoretical studies of compressible turbulence which have added considerably to knowledge in this area (see Lele 1994 for a review), but a convincing explanation of the reduced growth rate effect has been elusive.

A single compressibility parameter, the convective Mach number $M$, is sufficient to collapse experimental growth rate data to a reasonable accuracy, with growth rates normalized by the incompressible growth rate at the same density ratio. The convective Mach number was introduced by Bogdanoff (1983) and extensively used by Papamoschou \& Roshko (1988). For streams with equal ratio of specific heats we have $M=\left(U_{1}-U_{2}\right) /\left(c_{1}+c_{2}\right)$, where $U_{1}$ and $U_{2}$ are the two free-stream velocities and $c_{1}$ and $c_{2}$ are the free-stream sound speeds. A collection of the experimental results can be found in Lele (1994). One problem with the growth rate normalization is the lack of an accurate model for the incompressible growth rate as a function of density 
ratio, which leads to quite a large scatter in the normalized growth rates (Sandham \& Reynolds 1989; Lu \& Lele 1994). However, all the data show a large reduction in growth rate between $M=0.4$ and $M=0.8$. There is also evidence that the turbulence structure changes as $M$ increases. Flow visualizations (Clemens \& Mungal 1992, 1995) at high Reynolds numbers show how the low-Mach-number organized structure is lost as compressibility becomes important. Experiments by Elliot \& Samimy (1990) show that turbulence fluctuations and Reynolds stresses decrease as $M$ is increased.

In recent years, several explanations of compressibility effects have been offered. Zeman $(1990,1991)$ proposed that the dilatational part of the total dissipation became progressively important as the turbulent Mach number increased due to the appearance of eddy shocklets. He modelled dilatation dissipation as proportional to the solenoidal dissipation and a function of the turbulent Mach number $M_{t}$ and the kurtosis of the velocity field. By incorporating the model for dilatation dissipation into a second-moment closure formulation, he was able to obtain the growth rate reduction as $M$ increased. However, eddy shocklets have not been observed in experiment or direct numerical simulation below a convective Mach number of one (we exclude two-dimensional simulations, which have shown shocks above $M=0.7$ ) and thus there is no physical basis for dilatation dissipation being important in the key region of $M$ where the growth rate is significantly reduced. Sarkar et al. (1991) and El Baz \& Launder (1993) have also used models for dilatation dissipation to get the growth rate reduction. However, more recently, Sarkar (1995) has commented that there has been no direct validation of the concept for the mixing layer.

It has been observed that results for the growth rate of small disturbances in laminar compressible shear flow match the reduction in growth rate of the fully developed turbulent flow (see e.g. Sandham \& Reynolds 1990). This is a remarkable result and suggests that in some way the physical mechanisms in the turbulent flow are echoed in the linear regime. One would want an explanation of the reduced growth rate of mixing layers to be applicable to small disturbances as well as to the turbulent flow. This is certainly not the case for any theories based on changes in dissipation, as the linear results are obtained from inviscid stability theory, where the dissipation is zero.

Breidenthal (1990) proposed a sonic-eddy model for compressible turbulence based on the assumption that only eddies whose rotational Mach number $M_{r}$ is unity are efficient in entrainment. The rotational Mach number is based on the velocity difference across an eddy. The Kolmogorov spectrum of eddy scales would exist for all subsonic eddies $\left(M_{r}<1\right)$. According to this model, the normalized shear layer entrainment rate (growth rate) is a function of the largest rotational Mach number $M_{\delta}=\delta / \lambda=\Delta U / c$, where $\lambda$ is a sonic eddy size, $\delta$ the largest eddy scale, $\Delta U=U_{1}-U_{2}$ the velocity difference across the largest eddy and $c$ the speed of sound. A sudden drop in growth rate is predicted at $M_{\delta}=1$ when the largest eddies become sonic. Further transitions occur at very high Mach numbers when the turbulence microscale becomes equal to the mean free path and when the shear layer width becomes comparable with the mean free path. The model is conceptually important, but does not give quantitative information on growth rate or turbulence structure.

Up until very recently direct numerical simulation (DNS) of the compressible mixing layer had been limited to the early stages of vortex formation (Sandham \& Reynolds 1991). These revealed changes in typical eddy structure but did not contain small scales of turbulence. Recent work (Luo \& Sandham 1994, 1995; Vreman 1995; Vreman, Geurts \& Kuerten 1995a; Vreman, Kuerten \& Geurts 1995b) has succeeded in simulating compressible flows through a mixing transition to small-scale turbulence. In this paper we analyse turbulence data from these simulations to address 


\begin{tabular}{ccccccl}
$M$ & $R e$ & $L_{1}$ & $L_{2}$ & $L_{3}$ & Grid & \multicolumn{1}{l}{ Scheme } \\
0.2 & 50 & 59.0 & 59.0 & 59.0 & $192 \times 193 \times 192$ & finite difference \\
0.6 & 50 & 68.0 & 59.0 & 68.0 & $192 \times 193 \times 192$ & finite difference \\
0.8 & 140 & 26.7 & 60.0 & 26.7 & $144 \times 221 \times 160$ & Fourier/Padé \\
1.2 & 100 & 39.9 & 59.0 & 22.1 & $320 \times 513 \times 192$ & finite difference/upwind
\end{tabular}

TABLE 1. Specification of the DNS databases: Mach number, Reynolds number, computational box size, grid and spatial discretization.

the influence of compressibility on this building-block inhomogeneous flow problem. In $\$ 2$ we describe the DNS databases that were used in the study. The averaging procedure and statistical equations are given in $\$ 3$, and used to explain the growth rate reduction and anisotropy effects in $\S 4$. The results are discussed in $\S 5$.

\section{Direct numerical simulations}

The temporal mixing layer with equal and opposite free-stream velocities has been simulated by direct numerical simulation of the compressible Navier-Stokes equations. The database covers four different convective Mach numbers: $M=0.2,0.6,0.8$ and 1.2. Details of the $M=0.2,0.6$ and 1.2 cases are given in Vreman (1995) and Vreman et al. $(1995 a, b)$ and those of $M=0.8$ in Luo \& Sandham $(1994,1995)$. The Reynolds number, domain size, grid size and numerical scheme in each case are specified in table 1 . The Reynolds number is based on the reference values introduced below. In all cases the flow is simulated in a rectangular domain $\left[0, L_{1}\right] \times\left[-\frac{1}{2} L_{2}, \frac{1}{2} L_{2}\right] \times\left[0, L_{3}\right]$. The $x_{1}-, x_{2}$ - and $x_{3}$-coordinates correspond to the streamwise, normal and spanwise directions respectively. The boundary conditions in the homogeneous directions $\left(x_{1}\right.$ and $x_{3}$ ) are periodic, whereas the boundaries in the normal direction are treated with characteristic non-reflecting conditions in the $M=0.8$ case and as free-slip walls in the other cases.

The flow parameters have been non-dimensionalized with the (equal) free-stream values for density $\left(\rho_{\infty}\right)$, temperature and viscosity, half the free-stream velocity difference $\left(\frac{1}{2} \Delta U\right)$ and half the initial vorticity thickness $\left(\frac{1}{2} \Delta U /\left(\partial \bar{u}_{1} / \partial x_{2}\right)\right.$ in the centreplane). With this choice the free-stream pressure $\left(p_{\infty}\right)$ is non-dimensionalized by $\rho_{\infty}\left(\frac{1}{2} \Delta U\right)^{2}$. Hence after non-dimensionalization $\rho_{\infty}=1, \Delta U=2$ and $p_{\infty}=1 /\left(\gamma M^{2}\right)$, where $M=\frac{1}{2} \Delta U / c_{\infty}$ equals the standard convective Mach number for the case of equal free-stream sound speeds and $\gamma=1.4$ is the ratio of specific heats. The initial mean velocity profile in non-dimensional coordinates is prescribed by $u_{1}=\tanh \left(x_{2}\right)$. The non-dimensional density $\rho$, pressure $p$ and temperature $T$ are related by the equation of state $\rho T=\gamma M^{2} p$. The initial mean temperature is obtained with the CroccoBusemann relation, with uniform mean pressure. The Prandtl number is equal to one.

Perturbations obtained from linear stability theory are superimposed on the mean profile. The most unstable mode is two-dimensional for $M$ up to 0.6 , but threedimensional for higher $M$. The $M=0.2$ and 0.6 cases contain a two-dimensional disturbance to reflect the dominant instability and a three-dimensional disturbance to permit the transition to three-dimensional turbulence. The two-dimensional modes are $(4,0),(2,0)$ and $(1,0)$, where $(4,0)$ is the most unstable mode with wavelength equal to $L_{1} / 4$. The subharmonic modes $(2,0)$ and $(1,0)$ initiate vortex pairings. Three-dimensionality is included by adding the oblique mode disturbances 
$(4,4),(4,-4),(2,2),(2,-2),(1,1)$ and $(1,-1)$. Furthermore, random phase shifts in the oblique modes remove the symmetry in the initial conditions. For $M=0.8$ and 1.2 the primary instability is three-dimensional, so only a single pair of equal and opposite oblique modes is used. Their streamwise and spanwise wavelengths determine $L_{1}$ and $L_{3}$. In these cases the symmetry in the initial conditions is used to reduce computing time and storage requirements. The initial amplitude of the instability waves is small for $M=0.8(0.025)$, whereas the other simulations use large-amplitude disturbances (typically 0.1). If the initial amplitude is large, the Reynolds number can be slightly lower to trigger the transition to turbulence in a reasonably short time. The effect of Reynolds number on transition was addressed by Luo \& Sandham (1995).

Numerical details are also shown in table 1 . The table presents different numerical techniques for the several cases. Existing databases produced by different groups are combined in this work, which explains why the $M=0.2$ and 0.6 cases use a weighted fourth-order central difference scheme (Vreman et al. 1995a), whereas the $M=0.8$ case employs a Fourier collocation scheme in the periodic directions and a Padé scheme in the normal direction (Sandham \& Reynolds 1991; Luo \& Sandham 1994). The $M=1.2$ simulation presented here adopts the same numerical scheme as the $M=0.2$ and 0.6 cases if there are no shocks. However, when sufficiently strong shocks are detected, this scheme is replaced by a third-order upwind MUSCL scheme, which is more expensive than the original scheme, but is able to capture shocks (Vreman et al. 1995b). The shock is sufficiently strong if the absolute pressure gradient is above a critical value (see Vreman et al. 1995b). In each case the numerical method is fully explicit with Runge-Kutta integration in time. The accuracy of the simulations has been verified by grid refinement and in each case we present results for the finest grid. In the $M=0.8$ and 1.2 cases initial symmetries in the flow are exploited to decrease the computational cost. This does not affect the results. Coarser grid simulations without exploitation of the symmetry conditions demonstrated that the initial symmetry was preserved throughout the whole simulation.

The evolution of the momentum thickness $\delta$ (defined in the next section) for the cases considered is shown in figure 1. The later stages of the simulations are characterized by a strong growth of the momentum thickness, which is approximately linear with time. The growth rate $\delta^{\prime}$ equals the slope of the curve in this regime. The momentum thickness will only display exact linear growth if the mixing layer is self-similar. Self-similarity is hard to achieve with DNS since the computational domain in the homogeneous directions is finite. This reduces the statistical sample size and restricts the growth of large-scale structures close to the end of the simulations. Approximate values for the growth rate $\delta^{\prime}$ have been obtained from figure 1 for $\delta$ between 2 and 5 , giving growth rates of $0.072,0.058,0.038$ and 0.030 at $M=0.2$, $0.6,0.8$ and 1.2 respectively. The growth rate at $M=1.2$ is about $40 \%$ of its value at $M=0.2$ which is in broad agreement with the reduction found experimentally. Although fully self-similar flows have not been achieved, we can have confidence in the results, since the growth rate curve (symbols in figure 8) matches that found in experiments (symbols in figure 10).

In each simulation a mixing transition to small scales is observed, i.e. the breakdown of the large structures into small vortices. The scenario of the transition at $M=$ 0.2 is very close to that of the incompressible mixing layer. The two-dimensional instability leads to the formation of four rollers with spanwise vorticity. These rollers undergo pairing processes until finally one roller structure containing many small-scale structures fills the domain. The three-dimensional waves also grow and form $\Lambda$-shaped vortices and break down into small-scale motions. For the $M=0.6$ case the scenario 


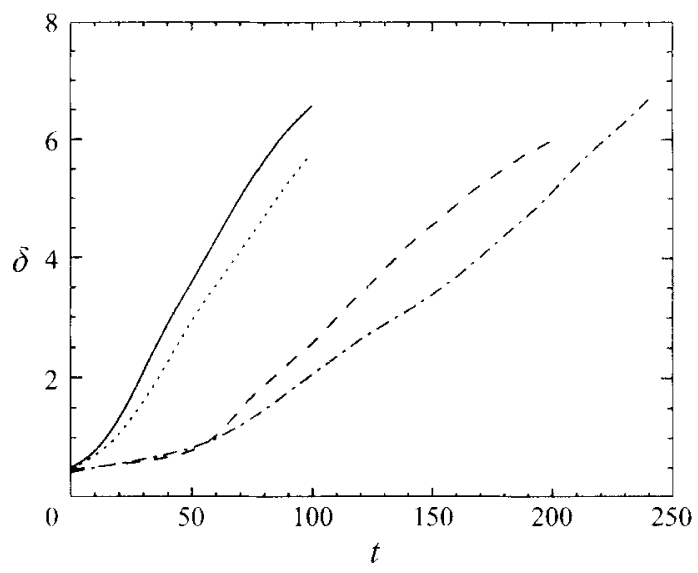

FIGURE 1. Graph of momentum thickness against time for Mach numbers $M=0.2$ (solid line), 0.6 (dotted), 0.8 (dashed), 1.2 (chain dotted).

is roughly the same, but the two-dimensional roller structure is less pronounced and can hardly be recognized anymore in the turbulent regime. In the $M=0.8$ case the primary three-dimensional instability forms $\Lambda$-shaped vortices, which initiate the mixing transition towards small scales. More details about the vortex structures in this simulation are given by Luo \& Sandham (1994). The structures in the simulation at $M=1.2$ are similar to those at $M=0.8$, but in addition eddy shocklets form (see below). As mentioned above, symmetries were imposed in the computations at $M=0.8$ and $M=1.2$, but this does not mean that the flow at these Mach numbers would not show turbulent behaviour. The flow is still turbulent, but with a symmetry imposed that would be broken only a long time later in a computation with no symmetry imposed. Others have also found that symmetries in the initial conditions persist even after mixing transition (Moser \& Rogers 1993).

Although we do not have exactly self-similar data, we do have a cascade to small scales and resolve the dissipation at these scales (which was checked by examination of energy spectra (Luo \& Sandham 1994; Vreman 1995; Vreman et al. 1995b). When turbulent quantities are averaged over a number of samples corresponding to different times we have a reasonable approximation to the statistics of a self-similar mixing layer.

In the simulation at $M=1.2$ shocks appear at three times: $t=122, t=182$ and $t=200$. Near $t=122$ and $t=182$ the shocks exist for only a few time units, but from $t=200$ to $t=225$ the flow is never free from shocks. These (rounded) times could accurately be determined by monitoring the maximum pressure gradient, since the appearance of a shock corresponds to a sudden increase of the maximum pressure gradient. Precise times and locations of the shocks are influenced by the domain sizes in the homogeneous directions and other flow parameters. In figure 2, pressure cuts through the computational domain show the locations of the shocks at the three representative times. Plots of dilatation have also been studied to confirm that the pressure gradients correspond to strong negative dilatation. No unique shock structure exists, since the shocks are all different in shape and orientation. At $t=122$ the shock is formed by supersonic values of the spanwise velocity and corresponds to large values of the pressure gradient in the spanwise direction. At $t=182$ and $t=200$ the shocks have curved shapes with large pressure gradients in the streamwise and normal 
(a)

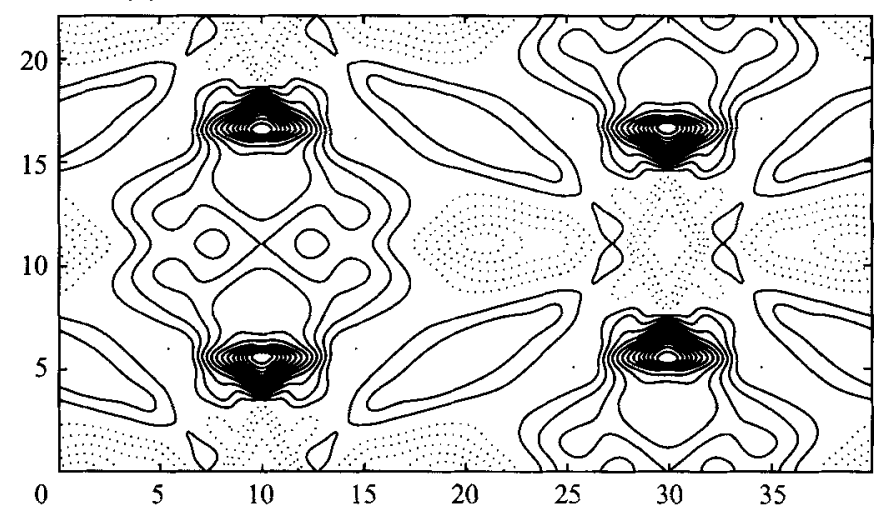

(b)

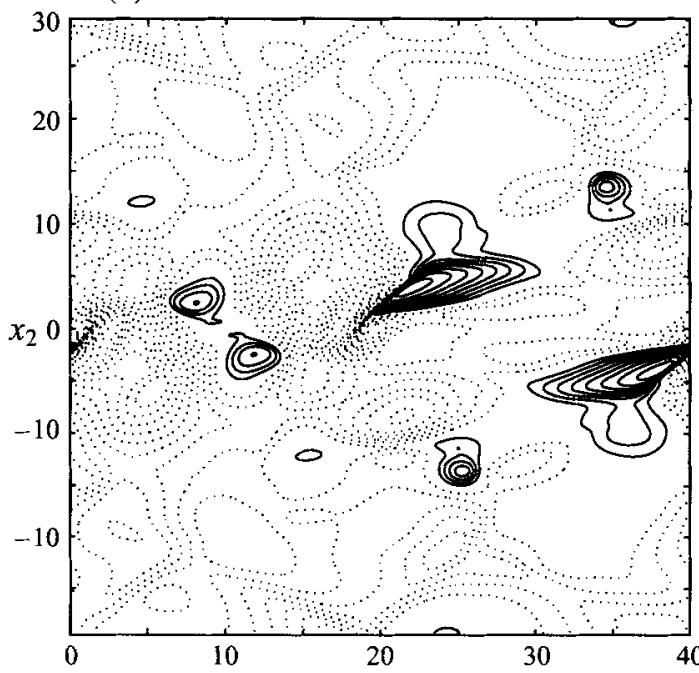

(c)

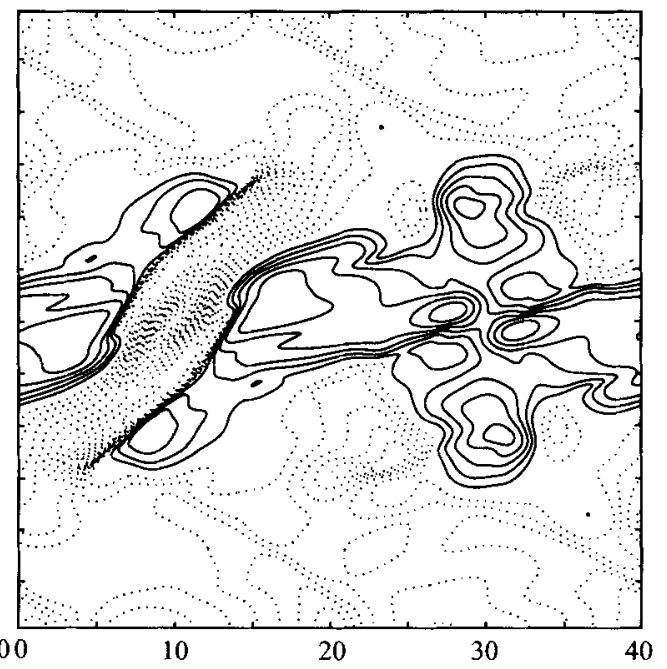

FIGURE 2. Slices through the pressure field showing the structure of eddy shock waves $\left(p<p_{\infty}\right.$ solid; $p>p_{\infty}$ dotted; contour increment 0.025): (a) $x_{2}=0$ slice at $t=122,(b) x_{3}=0$ slice at $t=182$ and (c) $x_{3}=3.9$ slice at $t=200$.

directions. At $t=182$, however, the velocity of the fluid passing through the shock is opposite to the mean flow velocity, whereas at $t=200$ it moves in the mean flow direction. As shocks had not been observed before in three-dimensional computations, we repeated the $M=1.2$ calculation with the compact finite difference/characteristic boundary condition code. Although the differences in the boundary conditions led to slightly different results, shock waves were still observed in the simulation. Further analysis of the eddy shock waves from the $M=1.2$ simulation is given in Vreman et al. (1995b). Some limited information on the existence of shocklets in laboratory flows has been presented by Papamoschou (1995).

\section{Data reduction and analysis}

In this section we use an analysis of integrated equations for the Reynolds stresses to study the changes in turbulence characteristics as the Mach number is increased. 
In $\$ 3.1$ we derive a new mathematical relation between growth rate and integrated turbulent production starting from the Reynolds-averaged Navier-Stokes equations. The integrated Reynolds stress transport equations are presented in $\$ 3.2$. We calculate the magnitude of each term from the DNS-databases and establish that the so-called dilatational terms are not important.

\subsection{The relation between growth rate and turbulent production}

The statistical description of turbulence is based on the Reynolds-averaged NavierStokes equations. In compressible flow the flow variables are written as

$$
\begin{aligned}
\rho & =\bar{\rho}+\rho^{\prime}, \\
p & =\bar{p}+p^{\prime}, \\
u_{i} & =\widetilde{u}_{i}+u_{i}^{\prime \prime} .
\end{aligned}
$$

In this notation $\bar{\rho}, \bar{p}$ and $\widetilde{u}_{i}$ represent the mean flow, whereas $\rho^{\prime}, p^{\prime}$ and $u_{i}^{\prime \prime}$ are the corresponding fluctuations. The basic averaging operator is the ensemble average, denoted with a bar. The tilde refers to Favre averaging, defined by $\widetilde{u}_{i}=\overline{\rho u}_{i} / \bar{\rho}$. The Reynolds-averaged continuity and momentum equations have the following form:

$$
\begin{aligned}
\frac{\partial \bar{\rho}}{\partial t} & =-\frac{\partial}{\partial x_{j}}\left(\bar{\rho} \widetilde{u}_{j}\right), \\
\frac{\partial}{\partial t}\left(\bar{\rho} \widetilde{u}_{i}\right) & =-\frac{\partial}{\partial x_{j}}\left(\bar{\rho} \tilde{u}_{i} \widetilde{u}_{j}\right)-\frac{\partial \bar{p}}{\partial x_{i}}+\frac{\partial \bar{\sigma}_{i j}}{\partial x_{j}}-\frac{\partial}{\partial x_{j}} \overline{\rho u_{i}^{\prime \prime} u_{j}^{\prime \prime}} .
\end{aligned}
$$

The last term in the momentum equation contains the Reynolds stress tensor, which results from averaging the nonlinear convective term. In a temporal shear layer the ensemble average (Reynolds average) is in practice performed as an average over the homogeneous directions, $x_{1}$ and $x_{3}$. The Reynolds-averaged variables are thus functions of $t$ and the normal coordinate $x_{2}$ only. This definition and the result derived in this subsection are not restricted to self-similar flow, in which the mean flow would be a function of $x_{2} / \delta(t)$ only, with $\delta(t)$ an appropriate thickness.

The momentum (or integral) thickness in a temporal compressible mixing layer is defined as

$$
\delta(t)=\frac{1}{\rho_{\infty}(\Delta U)^{2}} \int \bar{\rho}\left(U_{1}-\widetilde{u}_{1}\right)\left(\widetilde{u}_{1}-U_{2}\right) \mathrm{d} x_{2},
$$

where $\Delta U=U_{1}-U_{2}$. The integration in this formula is between the free-slip walls for the confined case, while it extends to infinity for the unconfined case. There is no unique way to define the thickness of a shear layer, but the thickness defined by equation (6) is generally regarded as an appropriate measure and has been widely used (Browand \& Latigo 1979; Ragab \& Sheen 1992; Rogers \& Moser 1994). An expression for the growth rate of the shear layer is obtained by differentiating equation (6):

$$
\delta^{\prime}=\frac{d \delta}{d t}=\frac{U_{1}+U_{2}}{\rho_{\infty}(\Delta U)^{2}} \int \frac{\partial\left(\bar{\rho} \widetilde{u}_{1}\right)}{\partial t} \mathrm{~d} x_{2}-\frac{1}{\rho_{\infty}(\Delta U)^{2}} \int \frac{\partial\left(\bar{\rho} \tilde{u}_{1} \tilde{u}_{1}\right)}{\partial t} \mathrm{~d} x_{2} .
$$

The first term at the right-hand side is zero as can be observed from the $x_{2}$-integrated mean momentum equation (5) for the streamwise direction. Since equation (5) is in conservative form, the right-hand side vanishes after integration in the $x_{2}$-direction if $\tilde{u}_{2}=0$ at the boundaries in the normal direction. With respect to the last term in 
equation (7), we consider the evolution of twice the mean flow kinetic energy, given by

$$
\frac{\partial}{\partial t}\left(\bar{\rho} \widetilde{u}_{1} \widetilde{u}_{1}\right)=\frac{\partial}{\partial x_{2}}\left(-\bar{\rho} \widetilde{u}_{1} \widetilde{u}_{1} \widetilde{u}_{2}+2 \bar{\sigma}_{12} \widetilde{u}_{1}-2 \overline{\rho u_{1}^{\prime \prime} u_{2}^{\prime \prime}} \widetilde{u}_{1}\right)+2 \overline{\rho u_{1}^{\prime \prime} u_{2}^{\prime \prime}} \frac{\partial \widetilde{u}_{1}}{\partial x_{2}}-2 \bar{\sigma}_{12} \frac{\partial \widetilde{u}_{1}}{\partial x_{2}},
$$

where $\bar{\sigma}_{12}$ is the viscous stress tensor. After integration the conservative terms vanish and hence the growth rate satisfies

$$
\delta^{\prime}=\frac{2}{\rho_{\infty}(\Delta U)^{2}}\left[\int\left(-\overline{\rho u_{1}^{\prime \prime} u_{2}^{\prime \prime}} \frac{\partial \widetilde{u}_{1}}{\partial x_{2}}\right) \mathrm{d} x_{2}+\int\left(\bar{\sigma}_{12} \frac{\partial \widetilde{u}_{1}}{\partial x_{2}}\right) \mathrm{d} x_{2}\right] .
$$

The first integral is the integrated turbulent production term, whereas the second integral represents the molecular dissipation of the mean flow. In the turbulent regime the latter can be neglected compared to the former and, consequently, the expression for the growth rate reduces to

$$
\delta^{\prime}=-\frac{2}{\rho_{\infty}(\Delta U)^{2}} \int\left(\overline{\rho u_{1}^{\prime \prime} u_{2}^{\prime \prime}} \frac{\partial \widetilde{u}_{1}}{\partial x_{2}}\right) \mathrm{d} x_{2} .
$$

Thus, the growth rate of time-developing turbulent mixing layers is proportional to the integrated turbulent production.

The relation between growth rate and production can be extended to spatial mixing layers under certain conditions. The convection speed in a spatial mixing layer is denoted by $U_{c}$ (equal to $\frac{1}{2}\left(U_{1}+U_{2}\right)$ for equal free-stream temperatures). We apply the following transformation: $x_{1}^{*}=x_{1}-U_{c} t, x_{2}^{*}=x_{2}, x_{3}^{*}=x_{3}$ and $t^{*}=t$. The NavierStokes equations are invariant under such a Galilean transformation. In this new frame of reference the spatial momentum thickness $\delta\left(x_{1}\right)$ can be written as $\delta^{*}\left(U_{c} t^{*}\right)$ and, consequently, the spatial growth rate satisfies

$$
\frac{\mathrm{d} \delta}{\mathrm{d} x_{1}}=\frac{1}{U_{c}} \frac{\mathrm{d} \delta^{*}}{\mathrm{~d} t^{*}}
$$

Equation (10) can be used for $\mathrm{d} \delta^{*} / \mathrm{d} t^{*}$ if we assume that terms with $\partial / \partial x_{1}^{*}$ are much smaller than terms with $\partial / \partial x_{2}^{*}$. This provides a relation between growth rate and integrated turbulent production for self-similar spatial mixing layers.

\subsection{The integrated Reynolds stress transport equations}

To exploit this connection between growth rate and integrated production we consider the $x_{2}$-integrated Reynolds stress transport equations. The Reynolds stress equations for compressible flow in their general form are given by Blaisdell, Mansour \& Reynolds (1991). The terms in divergence form vanish after integration, hence the equation for the $(i j)$-component of the integrated Reynolds stress becomes

$$
\frac{\mathrm{d}}{\mathrm{d} t} \int \overline{\rho u_{i}^{\prime \prime} u_{j}^{\prime \prime}} \mathrm{d} x_{2}=P_{i j}+\Pi_{i j}-\epsilon_{i j}
$$

where

$$
\begin{aligned}
P_{i j} & =-\int\left(\overline{\rho u_{i}^{\prime \prime} u_{2}^{\prime \prime}} \frac{\partial \widetilde{u}_{j}}{\partial x_{2}}+\overline{\rho u_{j}^{\prime \prime} u_{2}^{\prime \prime}} \frac{\partial \widetilde{u}_{i}}{\partial x_{2}}\right) \mathrm{d} x_{2}, \\
\Pi_{i j} & =\int p \overline{\left(\frac{\partial u_{i}^{\prime \prime}}{\partial x_{j}}+\frac{\partial u_{j}^{\prime \prime}}{\partial x_{i}}\right)} \mathrm{d} x_{2}, \\
\epsilon_{i j} & \left.=\int \overline{\left(\sigma_{i k} \frac{\partial u_{j}^{\prime \prime}}{\partial x_{k}}+\frac{\partial u_{i k}^{\prime \prime}}{\partial x_{k}}\right.}\right) \mathrm{d} x_{2},
\end{aligned}
$$


which represent the integrated production, pressure strain and dissipation tensors, respectively. Note that equation (10) implies

$$
P_{11}=\rho_{\infty}(\Delta U)^{2} \delta^{\prime}
$$

In a self-similar mixing layer the growth rate and, consequently, the integrated production is constant. Therefore, like Rogers \& Moser (1994), we argue that in a self-similar stage the terms of equation (12) are independent of time and scale with $\rho_{\infty}(\Delta U)^{3}$. Self-similarity also implies that the values of the Reynolds stress in the centre of the mixing layer have reached a constant level, whereas the width of the profiles grows with $\delta$. Therefore, we define

$$
R_{i j}=\frac{1}{\delta} \int \overline{\rho u_{i}^{\prime \prime} u_{j}^{\prime \prime}} \mathrm{d} x_{2},
$$

which will be independent of time in the self-similar stage and will scale with $\rho_{\infty}(\Delta U)^{2}$. Hence, the Reynolds stress equations reduce to an algebraic system

$$
\delta^{\prime} R_{i j}=P_{i j}+\Pi_{i j}-\epsilon_{i j}
$$

The values for the tensors $R_{i j}, P_{i j}, \Pi_{i j}$ and $\epsilon_{i j}$ have been plotted against Mach number in figure 3. Since we do not have exact self-similarity over time, the values in figure 3 have been averaged over a number of samples in the region with approximately linear growth of the momentum thickness (figure 1). The contributions of the $(1,3)$ and $(2,3)$ components of the Reynolds stress have not been plotted since they are negligible compared to the other components. Figure 3(a) shows that the anisotropy of the diagonal Reynolds stresses increases with Mach number. The Reynolds shear stress $R_{12}$ is directly coupled to the growth rate through $P_{11}$ (see (10)) and decreases with Mach number. Its anisotropy $\left(R_{12} / R_{q q}\right)$ is almost constant with increasing Mach number. From figure 3(b), comparison of the production term $P_{11}$ with the growth rates obtained from figure 1 illustrates equation (16): $P_{11}=4 \delta^{\prime}$. The production terms $P_{22}$ and $P_{33}$ are zero according to figure $3(b)$, which is expected since the only significant mean velocity derivative, $\partial \tilde{u}_{1} / \partial x_{2}$, does not appear in these terms. Retaining only the significant terms, the system given by (18) and (16) reduces to

$$
\begin{array}{lr}
\delta^{\prime} R_{11}=\rho_{\infty}(\Delta U)^{2} \delta^{\prime}+ & \Pi_{11}-\epsilon_{11}, \\
\delta^{\prime} R_{22}= & \Pi_{22}-\epsilon_{22}, \\
\delta^{\prime} R_{33}= & \Pi_{33}-\epsilon_{33}, \\
\delta^{\prime} R_{12}= & P_{12}+\quad \Pi_{12}-\epsilon_{12} .
\end{array}
$$

The pressure-strain term $\Pi_{11}$ is negative, while $\Pi_{22}$ and $\Pi_{33}$ are positive. Thus, pressure-strain acts to redistribute energy from the streamwise into the normal and spanwise fluctuations. We remark that the pressure dilatation $\frac{1}{2} \Pi_{k k}$ is approximately zero, even at the highest $M$.

The dissipation component $\epsilon_{11}$ does not decrease as much as the other two components as the Mach number is increased. Thus, the anisotropy of the diagonal dissipation components increases at higher Mach numbers. The diagonal components of the dissipation tensor are considerably larger than $\epsilon_{12}$, although the latter is not zero. Tennekes \& Lumley (1972) argue that for very high Reynolds numbers the turbulence is isotropic at small scales, which would imply isotropic dissipation. Our simulations have all been conducted at low Reynolds numbers, and it may be that the turbulent cascade is not over a sufficiently wide range of scales to set up isotropy 

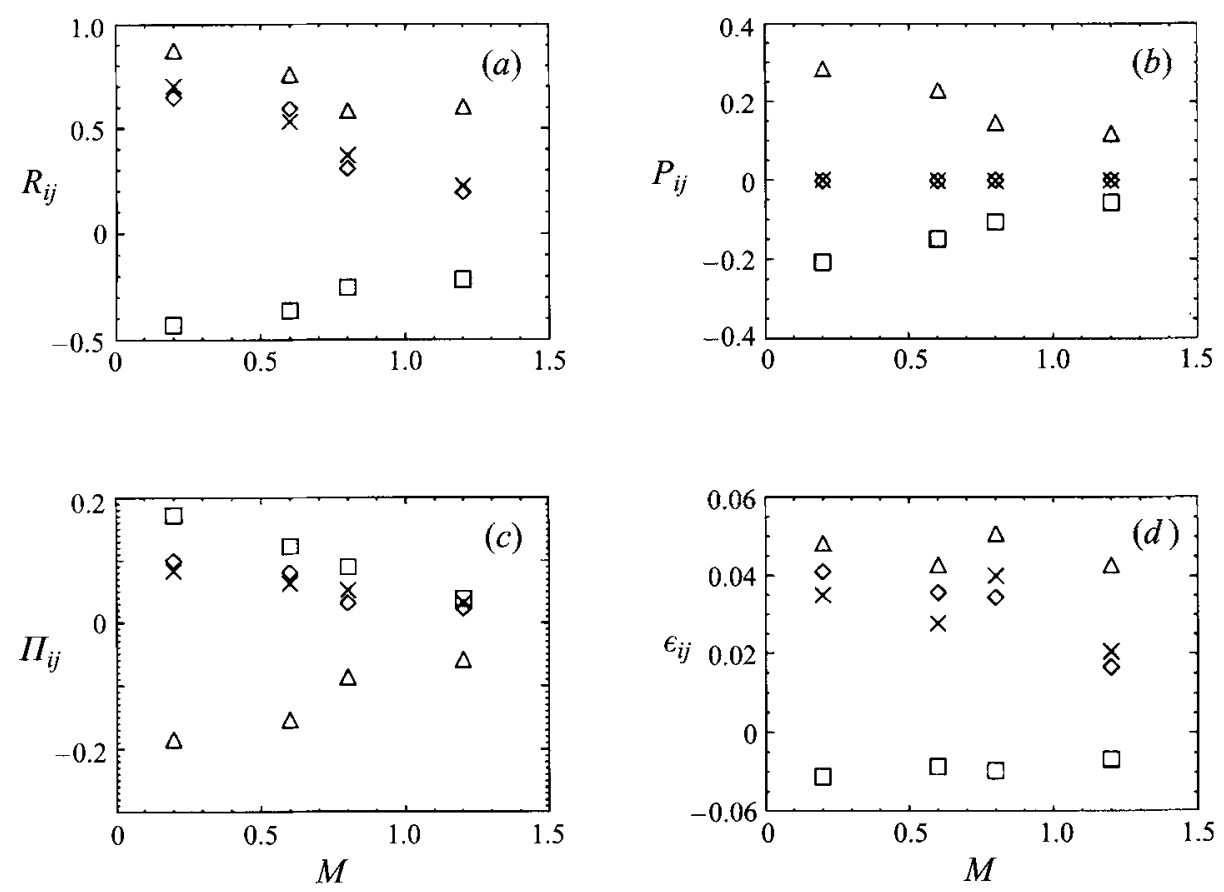

FigURE 3. Integrated turbulence statistics: (a) Reynolds stress $R_{i j},(b)$ production $P_{i j}$, (c) pressure-strain $\Pi_{i j}$ and $(d)$ dissipation $\epsilon_{i j}$. Components are denoted with triangles (11), crosses (22), diamonds (33) and squares (12).

in the dissipation. However, a tendency to isotropy of the small scales is observed in the simulations, since the anisotropy of the diagonal dissipation is smaller than that of the diagonal Reynolds stresses.

Dilatation dissipation and pressure-dilatation are of interest because of their possible use in explaining compressibility effects. Total dissipation $\epsilon$ can be split as $\epsilon=\epsilon_{s}+\epsilon_{d}$, where $\epsilon_{s}$ is a solenoidal part and $\epsilon_{d}$ is a dilatational part. An integrated form of the dilatational dissipation is

$$
\epsilon_{d}=\int \frac{4}{3} \frac{\widetilde{\mu}}{\operatorname{Re}} \overline{\frac{\partial u_{i}^{\prime \prime}}{\partial x_{i}} \frac{\partial u_{j}^{\prime \prime}}{\partial x_{j}}} \mathrm{~d} x_{2}
$$

We consider the highest Mach number case $M=1.2$ and in figure 4 show the integrated total dissipation, dilatation dissipation and pressure-dilatation as functions of time. The dilatational part of the dissipation is very small even in the stages of the simulation which contain eddy shocklets. The pressure-dilatation is somewhat larger, but it changes sign a number of times and is also not significant compared to the total dissipation. These two dilatational terms are even smaller in the cases with lower convective Mach number which do not contain eddy shocklets. Eddy shocklets do increase the dilatational terms, but compared to the total dissipation their contributions are too small to explain the growth rate reduction. The production and pressure-strain rate terms are much more affected by compressibility than the dissipation and, consequently, the reduced growth rate must be explained from these terms.

The production and pressure-strain terms in the $R_{11}$ and $R_{12}$ equations have opposite signs. However, the reduced growth rate is not explained by an increase in 


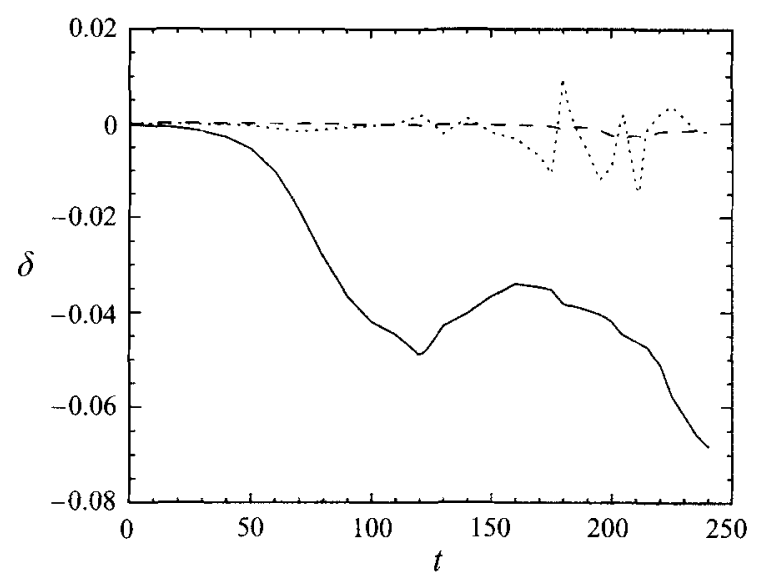

FIGURE 4. Comparison of the magnitudes of total dissipation (solid line) with dilatation-dissipation (dashed line) and pressure-dilatation (dotted line) during the simulation at $M=1.2$.

the ratio of pressure-strain and production in these equations. Both production and pressure-strain terms reduce strongly with Mach number. The ratio also reduces, but only slightly. In the following section we explain the growth rate reduction from the diagonal Reynolds stress equations, since the growth rate was found to be directly connected to the production term in one of these equations.

\section{Modelling the effect of compressibility}

In this section we identify the key terms contributing to the reduced growth rate and build a complete model for the integrated diagonal Reynolds stress equations using a deterministic model for pressure fluctuations and models for turbulence anisotropy. In $\$ 4.1$ we argue that the mixing layer growth rate would be proportional to the rapid pressure- strain term if the turbulence were isotropic (i.e. $R_{11}=R_{22}=R_{33}$ ). Furthermore, the rapid pressure strain term is expressed in the pressure extrema. In $\$ 4.2$, we model the pressure extrema as functions of the convective Mach number, using a compressible vortex model and the sonic-eddy concept. Thus, the isotropic model is completed, which gives a good qualitative prediction of the growth rate reduction. However, it predicts too low growth rates at high Mach numbers. In $\S 4.3$ we correct the isotropic model taking anisotropy effects into account to obtain a better quantitative prediction of the growth rate reduction.

\subsection{The significance of pressure-strain}

In the following subsections we explain the reduced growth rate of the mixing layer by consideration of a necessary adjustment in pressure fluctuations as the Mach number is increased. A reformulation in terms of anisotropy is useful. We define the Reynolds stress anisotropy as

$$
b_{i j}=\frac{R_{i j}-\frac{2}{3} k \delta_{i j}}{2 k}
$$

and the dissipation anisotropy as

$$
e_{i j}=\frac{\epsilon_{i j}-\frac{2}{3} \epsilon \delta_{i j}}{2 \epsilon}
$$




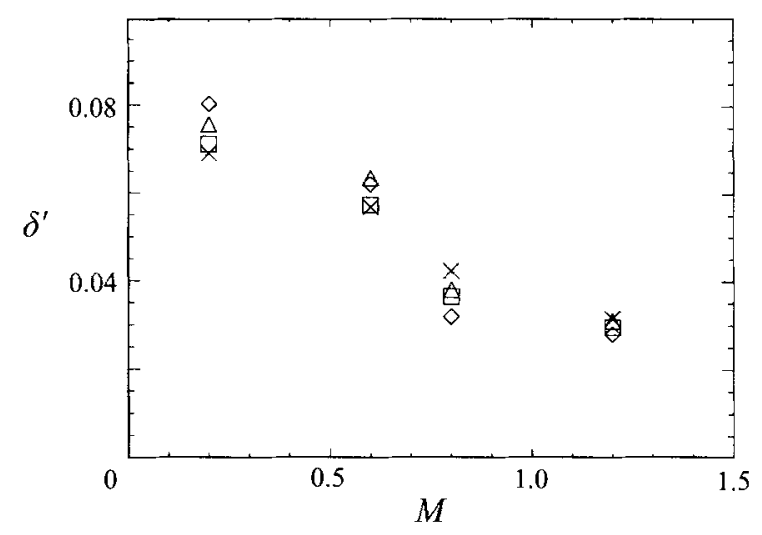

FiguRE 5. The growth rate $\delta^{\prime}$ obtained from equation (26) (triangles), equation (27) (crosses) and equation (28) (diamonds) compared to the growth rate obtained from figure 1 (squares).

where $k=R_{q q} / 2$ and $\epsilon=\epsilon_{q q} / 2$ are respectively the turbulence kinetic energy and dissipation. With the assumption that we can neglect the pressure-dilatation term in the kinetic energy equation, the integrated equations for the diagonal terms reduce to

$$
\begin{aligned}
& \delta^{\prime}\left(2 b_{11} k-\frac{2}{3} \rho_{\infty}(\Delta U)^{2}\right)=\Pi_{11}^{S}+\Pi_{11}^{R}-2 e_{11} \epsilon, \\
& \delta^{\prime}\left(2 b_{22} k+\frac{1}{3} \rho_{\infty}(\Delta U)^{2}\right)=\Pi_{22}^{S}+\Pi_{22}^{R}-2 e_{22} \epsilon, \\
& \delta^{\prime}\left(2 b_{33} k+\frac{1}{3} \rho_{\infty}(\Delta U)^{2}\right)=\Pi_{33}^{S}+\Pi_{33}^{R}-2 e_{33} \epsilon,
\end{aligned}
$$

where we have included a split of the pressure-strain into a slow part $\Pi^{S}$ and a rapid part $\Pi^{R}$ (see e.g. Speziale, Sarkar \& Gatski 1991). In incompressible flow the slow part is associated with the process of return to isotropy, while the rapid part is associated with the mean strain rate. Extension to compressible flow is an area of current research. The recent work of Ristorcelli (1995) is an example of the distinction between slow and rapid parts in compressible flow. Another approach is to consider the split to be between a linear part (predictable in principle by rapid distortion theory) and a nonlinear part (see e.g. Cambon, Coleman \& Mansour 1993). In this paper the part of pressure-strain responsible for the return to isotropy is simply defined as the slow part, whereas the remainder is called the rapid part.

Equations (26) to (28) can be used to measure the deviation from self-similarity of the simulation data. In figure 5 the growth rates $\delta^{\prime}$ versus $M$ calculated from these equations have been plotted together with the growth rates obtained from figure 1 . The maximum difference between $\delta^{\prime}$ obtained from equation (26) and $\delta^{\prime}$ from figure 1 is $9 \%$, whereas the maximum differences are $10 \%$ for equation (27) and $16 \%$ for equation (28). These deviations indicate that the simulated flows are not fully self-similar. The deviation is sufficiently small to permit the use of the simulation data (in particular for equation (26), the main basis of our analysis).

The diagonal-pressure strain terms mainly determine the growth rate as is shown by the relative magnitude of the terms in equations (26) to (28). The Reynolds stress anisotropy term $2 b_{11} k$ is usually small compared to $\frac{2}{3} \rho_{\infty}(\Delta U)^{2}$. Compared to pressure-strain the dissipation anisotropy term is not large either and is assumed to vanish for very high Reynolds number (Tennekes \& Lumley 1972). This results in an approximate proportionality between the growth rate and the diagonal pressurestrain components, which is confirmed by the data (figure 3 ). If the turbulence were isotropic the diagonal components of $b_{i j}$ and $\Pi_{i j}^{S}$ would vanish and equations (26) to 


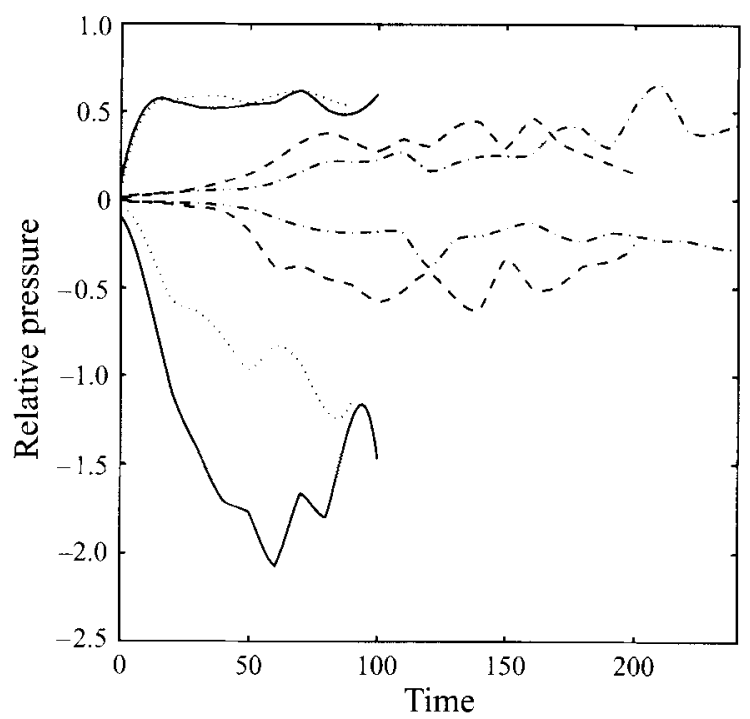

FIGURE 6. Maximum and minimum pressures relative to free-stream pressure during simulations at $M=0.2$ (solid), 0.6 (dotted), 0.8 (dashed) and 1.2 (chain dotted), showing the large reduction of pressure depressions as Mach number is increased.

(28) would reduce to an exact proportionality between the rapid pressure-strain term and the mixing layer growth rate:

$$
\delta^{\prime}=-\frac{3}{2 \rho_{\infty}(\Delta U)^{2}} \Pi_{11}^{R} .
$$

The relation between the diagonal components of pressure-strain would be $\Pi_{22}^{R}=$ $\Pi_{33}^{R}=-\frac{1}{2} \Pi_{11}^{R}$.

It is proposed that the main effect of compressibility comes from modified pressure fluctuations. That the pressure fluctuation must change is evident when one considers that the typical pressure fluctuations in incompressible flow, normalized by $\rho_{\infty}\left(\frac{1}{2} \Delta U\right)^{2}$ would lead to negative pressures in high-Mach-number flow, since the free-stream pressure drops relative to $\rho_{\infty}\left(\frac{1}{2} \Delta U\right)^{2}$ as $1 /\left(\gamma M^{2}\right)$. This is illustrated by the simulation data in figure 6, where the relative pressure extrema $p^{+}=p_{\max }-p_{\infty}$ and $-p^{-}=$ $-\left(p_{\infty}-p_{\min }\right)$ are plotted against time. The reference pressure $p_{\infty}$ equals $16.85,1.98$, 1.12 and 0.50 for the different Mach numbers respectively. A large drop in $p^{-}$is observed. Since the values for $p^{-}$in the nearly incompressible case $(M=0.2)$ are considerably larger than $p_{\infty}$ at $M=1.2, p^{-}$must decrease with increasing Mach number in order to keep the minimum pressure positive.

The reduced pressure fluctuations cause the total pressure-strain terms to reduce and the rapid components in particular. Thus, we propose to model $\Pi_{11}^{R}$ as

$$
\Pi_{11}^{R}=p^{*}(M)\left(\Pi_{11}^{R}\right)_{M=0}
$$

where $p^{*}(M)$ contains the effect of reduced pressure fluctuations. In the isotropic approximation the mixing layer growth rate would be proportional to $p^{*}(M)$.

To estimate $p^{*}(M)$ we assume that

$$
p^{*}(M)=\frac{\left(p_{\max }-p_{\min }\right)_{M}}{\left(p_{\max }-p_{\min }\right)_{0}}
$$


where $p_{\max }$ and $p_{\min }$ will be estimated from a deterministic model of large vortex structures in compressible shear flow, assumed to be representative of the typical eddies that contribute to the rapid pressure-strain term.

\subsection{Deterministic model for pressure extrema}

Pressure minima in a flow can be identified with the cores of vortices, whereas pressure maxima occur at stagnation points. We present a model for the pressure minima first. A common description of a region of rotating fluid is the Oseen vortex, which has been used before to represent vortices in mixing layers (Papamoschou \& Lele 1992). It assumes axisymmetric flow with the tangential velocity given by

$$
v_{\theta}=\frac{\Gamma}{2 \pi r}\left(1-\mathrm{e}^{-\alpha r^{2} / R^{2}}\right),
$$

where $\Gamma$ is the circulation, $R$ is the vortex size, and $\alpha=1.256$ is chosen such that $v_{\theta}=v_{\theta, \max }$ at $r=R$.

In order to obtain the pressure minimum we turn to the inviscid radial momentum equation:

$$
\frac{1}{\rho} \frac{\partial p}{\partial r}=\frac{v_{\theta}^{2}}{r} .
$$

To include the effects of density variation on the pressure field inside a vortex we need a model for the thermodynamics. We observe from two-dimensional simulations (Sandham \& Reynolds 1989) at $M=0.6$ that the temperature changes inside vortices developing in temporal mixing layers are only $7 \%$ relative to the free stream, compared with fluctuations of $40-50 \%$ for the density and pressure. With the assumption of isothermal flow we can integrate (33) to get

$$
\frac{p_{\min }}{p_{\infty}}=\exp -\left(\gamma A v_{\theta, \max }^{2} / c_{\infty}^{2}\right),
$$

where $c_{\infty}=\left(\gamma p_{\infty} / \rho_{\infty}\right)^{1 / 2}$ is the free-stream speed of sound and

$$
A=\frac{1}{v_{\theta, \max }^{2}} \int_{0}^{\infty} \frac{v_{\theta}^{2}}{r} \mathrm{~d} r .
$$

We assume that $A$ is a constant, equal to 1.69 for the Oseen vortex.

Although the Oseen vortex is two-dimensional and inviscid it can provide a reasonable description of the pressure drop inside a vortex. A more general vortex is the Burgers vortex which is a three-dimensional solution of the viscous Navier-Stokes equations and has served as an illustration of the vortex stretching mechanism in turbulence (Burgers 1948). The tangential velocity is again given by equation (32), with $\alpha / R^{2}=V / 2 v$. The other two velocity components are $v_{r}=-V r$ and $v_{z}=2 V z$. However, for a typical vortex radius $(R), V$ turns out to be quite small for small $v$ (high Reynolds number). The corresponding pressure correction to equation (34), proportional to $V^{2}$, is then negligible.

The pressure drop is not strongly influenced by the precise equation of state either. We have chosen an isothermal vortex, since in the inner region of turbulent vortices the entropy is increased. The pressure minimum, however, would only change slightly with the assumption of isentropic flow in the vortex core.

The pressure drop in equation (34) is then a function of the maximum tangential velocity in a vortex, $v_{\theta, \max }$. A logical value for $v_{\theta, \max }$ at low Mach numbers is half the 
difference between the free-stream velocities,

$$
v_{\theta, \max }=\frac{1}{2} \Delta U
$$

which gives rise to a pressure drop of $p_{\min }-p_{\infty}=-A \rho_{\infty}\left(\frac{1}{2} \Delta U\right)^{2}$ for incompressible mixing layers (obtained from (34) for $M \rightarrow 0$ ). For compressible flows we consider the rotational Mach number based on the velocity difference across the eddy,

$$
M_{r}=\frac{2 v_{\theta, \max }}{c_{\infty}}=\frac{4 v_{\theta, \max }}{\Delta U} M
$$

where $M$ is the convective Mach number. If equation (36) were used for all Mach numbers, $M_{r}$ would become larger than unity for $M>0.5$. However, the conceptual 'sonic-eddy' model of Breidenthal (1990) implies that only eddies with $M_{r}<1$ play a role in the turbulent energy cascade in a compressible flow. It is therefore proposed that the following model be used for $v_{\theta, \max }$ :

$$
v_{\theta, \max }=\min \left(1, \frac{M_{\text {crit }}}{M}\right) \frac{1}{2} \Delta U .
$$

$M_{c r i t}$ is the critical Mach number beyond which $v_{\theta, \max }$ is affected by compressibility. The value $M_{\text {crit }}=0.5$ corresponds to the sonic-eddy model in which the largest eddies satisfy $M_{e}=1$. Without the sonic-eddy model we find that the pressure in the vortex cores would drop down to almost zero pressure, which is not observed in the simulation results.

Next we turn to the prediction of the pressure maxima which correspond to stagnation points. Isentropic flow is a good approximation for the fluid on a streamline towards a stagnation point and the standard relation yields

$$
\frac{p_{\max }}{p_{\infty}}=\left(1+\frac{1}{2}(\gamma-1) M^{2}\right)^{\gamma /(\gamma-1)}
$$

Above $M_{\text {crit }}$ we use the above formula with $M=M_{\text {crit }}$, which corresponds to flow stagnating around sonic eddies. For incompressible mixing layers the pressure rise $p_{\max }-p_{\infty}$ equals $\frac{1}{2} \rho_{\infty}\left(\frac{1}{2} \Delta U\right)^{2}$. This value is obtained if the limit $M \rightarrow 0$ is taken and is also predicted by Bernoulli's equation. Physically, the incompressible limit does not necessarily mean that the velocity difference is reduced to zero; it can also mean that the speed of sound tends to infinity.

The results for the pressure variations are summarized in figure 7 , obtained by employing equations (34) and (38) for $p_{\min }$ and (39) for $p_{\max }$. Smoothing has been applied to the pressure data to remove the discontinuity in the slope at $M_{\text {crit }}$. This only affects the curves in the immediate vicinity of $M_{\text {crit }}$. Figure $7(a)$ shows pressure relative to free-stream pressure plotted against Mach number. The solid lines are the pressure in the core of the vortex and the pressure at the stagnation point (further discussed in $\$ 5$ ). The dashed line shows the curve for zero pressure. It can be seen how both the core and stagnation pressures reduce as the Mach number is increased to satisfy the constraint that absolute pressure cannot drop below zero. If $p_{\min }-p_{\infty}$ kept its incompressible value, we would have negative absolute pressure at $M=0.65$.

Figure $7(b)$ shows the parameter $p^{*}(M)$ defined by equation (31), which is equal to the growth rate reduction if the Reynolds stress and dissipation are isotropic. A simple qualitative explanation for the growth rate reduction is that growth rate is proportional to pressure-strain and the pressure fluctuations must reduce as the Mach number is increased to avoid negative pressures. 

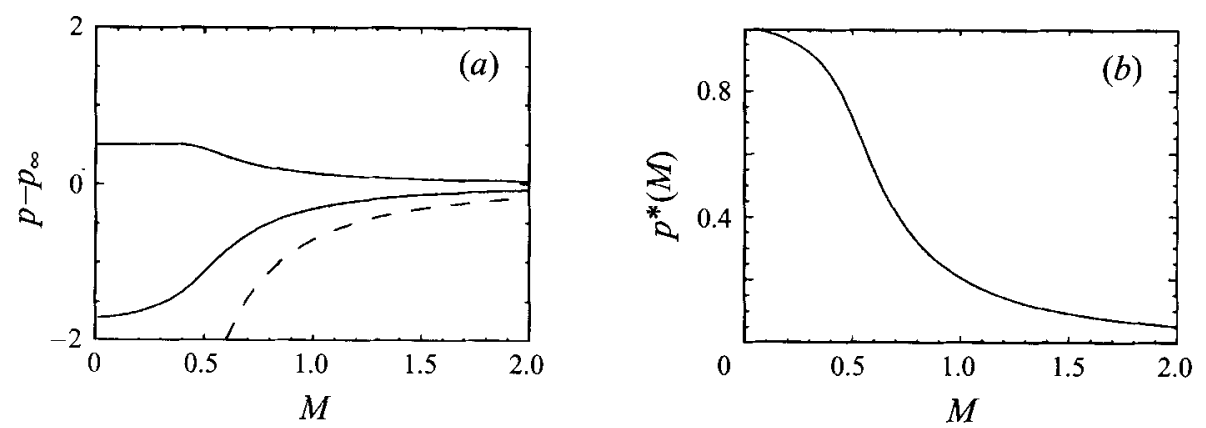

FiguRE 7. Results of the pressure model: (a) pressure maximum and minimum (solid lines) relative to the free-stream pressure, also showing the lowest possible pressure corresponding to zero pressure in the vortex cores (dashed line); $(b)$ the pressure reduction function $p^{*}(M)$ showing the strong reduction with increasing Mach number for eddies contributing to the rapid part of the pressure strain.

\subsection{Anisotropy effects}

We have seen that a simple isotropic picture of turbulence in the compressible mixing layer is sufficient to explain qualitatively the growth rate reduction. At higher $M$ in particular, the isotropic picture underpredicts the growth rate and needs correction. For quantitative predictions we need therefore to consider the anisotropy of the turbulence. In this section we model all the terms in the integrated equations for the diagonal Reynolds stresses in order to get a closed form for the growth rate. We need to distinguish clearly where approximations are made. We regard the following as accurate and well supported by the data: $(a) P_{11}=\rho_{\infty}(\Delta U)^{2} \delta^{\prime}$, as derived in $\S 3.1$, and $P_{22}=P_{33}=0 ;(b)$ negligible pressure dilatation, and hence from the turbulence kinetic energy equation $\epsilon=\delta^{\prime}\left(\frac{1}{2} \rho_{\infty}(\Delta U)^{2}-k\right)$; and (c) negligible dilatational dissipation.

Our main model equation is (26), using the pressure reduction function (31) on the rapid pressure-strain and the usual Rotta form for the slow pressure-strain term. Isotropic dissipation is still assumed $\left(e_{11}=0\right)$. Thus,

$$
\delta^{\prime}\left(2 b_{11} k-\frac{2}{3} \rho_{\infty}(\Delta U)^{2}\right)=-2 c_{1} \epsilon b_{11}-c_{2} p^{*}(M) \rho_{\infty}(\Delta U)^{3} .
$$

To reduce this equation to a closed equation for $\delta^{\prime}$, it is sufficient to express the anisotropy $b_{11}$ and the Reynolds stresses $R_{22}$ and $R_{33}$ in terms of $\delta^{\prime}$. We suggest the following models:

$$
\begin{aligned}
& b_{11}=\left(b_{11} \delta^{\prime}\right)_{M=0} / \delta^{\prime}=c_{3} \Delta U / \delta^{\prime}, \\
& R_{22}=R_{33}=\left(R_{33} / \delta^{\prime}\right)_{M=0} \delta^{\prime}=c_{4} \rho_{\infty} \Delta U \delta^{\prime},
\end{aligned}
$$

expressing that the anisotropy of the diagonal stresses increases if the growth rate decreases. The anisotropy of the turbulence is expected to change, since there is a move to more streamwise turbulence structures as the Mach number is increased. By analogy with other flows where streamwise structure becomes important we expect the anisotropy $b_{11}$ to increase. The precise functional form of $b_{11}, R_{22}$ and $R_{33}$ is hypothetical and other choices could be equally or more plausible. However, the models above are in reasonable agreement with the data and lead to a relatively simple equation for $\delta^{\prime}$.

As model constants we use $c_{1}=2.2$ and $c_{2}=0.021, c_{3}=0.0025$ and $c_{4}=4.5$. The Rotta constant $c_{1}$ is the same as in some second-moment closures (Gibson \& 


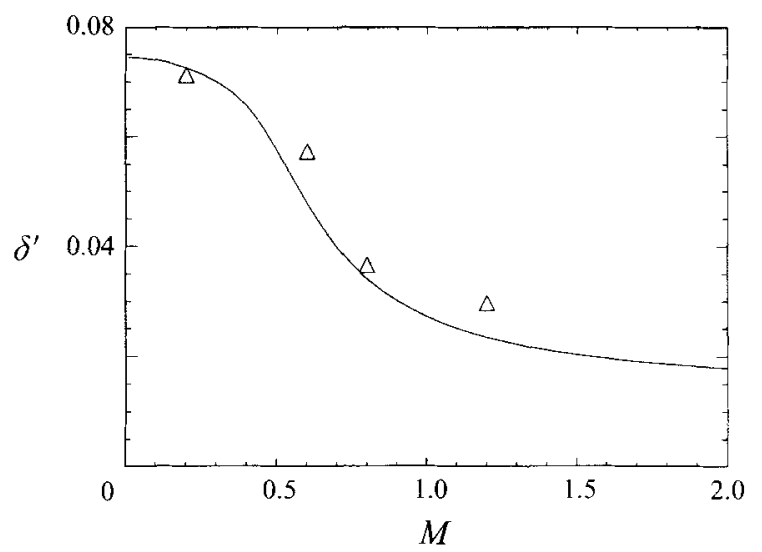

FIGURE 8. Graph of the model prediction for growth rate $\delta^{\prime}$ (solid line), compared with simulation data (symbols).

Launder 1978). All other constants are estimated for incompressible flow (roughly equal to the $M=0.2$ simulation results).

Putting all of the above together, we can derive a quadratic equation for the growth rate $a\left(\delta^{\prime} / \Delta U\right)^{2}+b \delta^{\prime} / \Delta U+c=0$ where the coefficients are related to the model constants by

$$
\begin{aligned}
& a=\frac{4}{9}+2 c_{3} c_{4}\left(c_{1}-1\right), \\
& b=-\frac{2}{3} c_{3}\left(1+c_{1}\right)-\frac{2}{3} c_{2} p^{*}(M), \\
& c=c_{2} c_{3} p^{*}(M)+c_{1} c_{3}^{2} .
\end{aligned}
$$

Only the largest root of the quadratic is realizable (positive $\epsilon$ ) and once $\delta^{\prime}$ is known all other quantities can be easily found.

Figures 8 and 9 give the predictions from the model up to $M=2$ (with $\rho_{\infty}=1$, $\Delta U=2$ ), compared with the simulation data. Figure 8 shows the growth rate against $M$ compared with the simulation data. The behaviour for larger Mach numbers is of considerable interest, but we should caution that there is little experimental or numerical data available between $M=1.2$ and $M=2$ to validate the model. Figure 9 shows various turbulence quantities. The Reynolds stresses (figure $9 a, b$ ) are in good agreement with the simulation results. The $R_{22}$ and $R_{33}$ components decrease proportional to the growth rate, while the $R_{11}$ component levels out at about half its incompressible level. Figures $9(c)$ and $9(d)$ show the pressure-strain term and Reynolds stress anisotropy $b_{11}$ compared with simulation data. The pressure-strain term especially is in good agreement with the simulation data. Turbulence kinetic energy $k$ and dissipation $\epsilon$ are shown in figures $9(e)$ and $9(f)$. The dissipation is unaffected by compressibility at low Mach numbers and only decreases by about a factor of two up to $M=2$.

The deviations from the isotropic picture are most significant at high Mach numbers, where in the anisotropic model a larger growth rate is found than in the isotropic case. The isotropic model predicts zero growth rate for infinite Mach number, since the growth rate is proportional to $p^{*}(M)$. The limiting growth rate for $M \rightarrow \infty$ provided by the anisotropic model is obtained if $p^{*}(M)=0$ is substituted in the coefficients of the quadratic (43)-(45). Using the values of the model constants proposed above, we obtain $\delta^{\prime} \rightarrow 0.015$ for $M \rightarrow \infty$, which is about $20 \%$ of the incompressible 

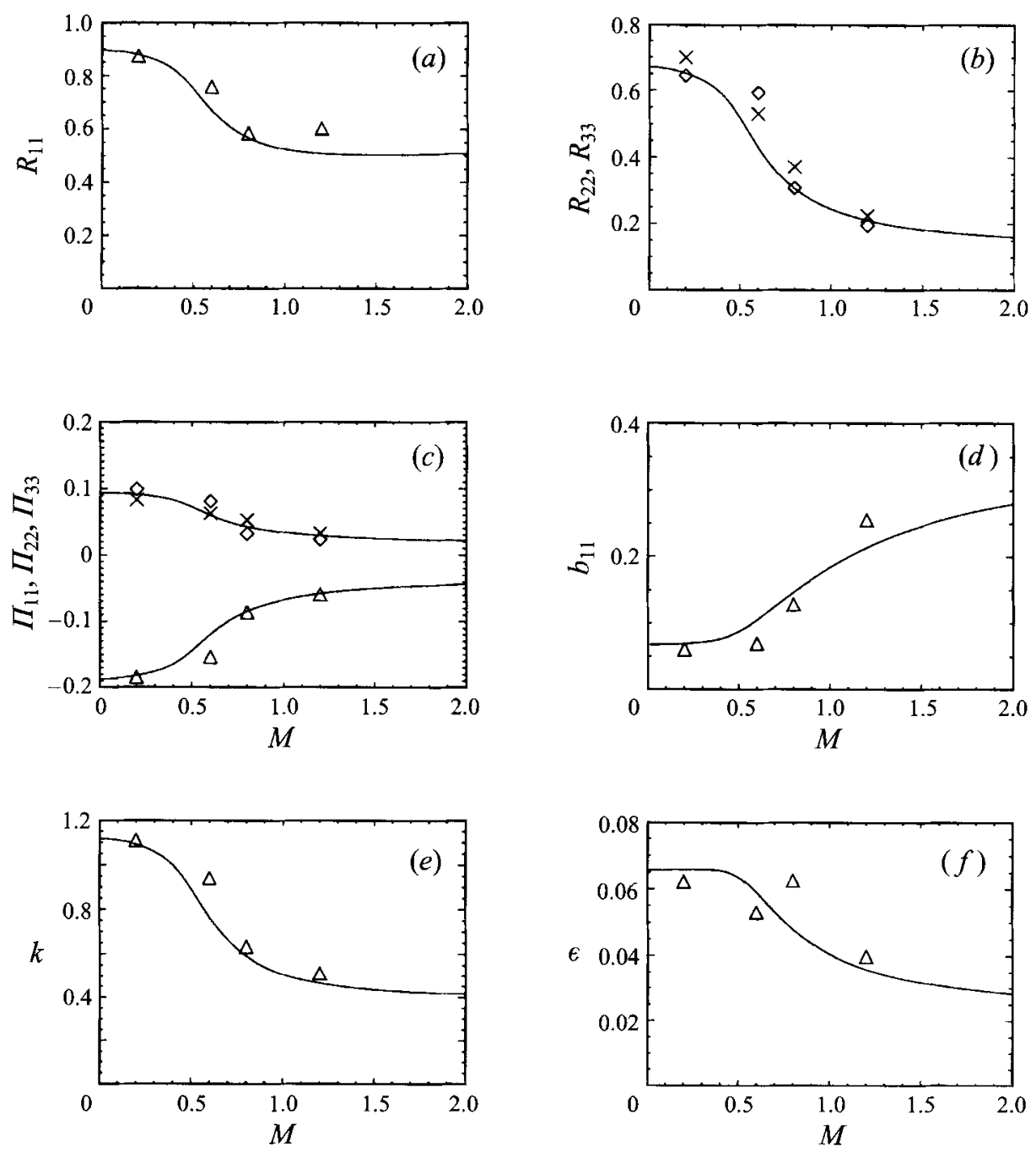

FIGURE 9. Turbulence statistics from model (solid lines) compared with simulation data (triangles for 11-component, crosses for 22, diamonds for 33): (a) Reynolds stress $R_{11}$, (b) Reynolds stresses $R_{22}$ and $R_{33},(c)$ pressure strain $\Pi_{11}, \Pi_{22}$ and $\Pi_{33},(d)$ Reynolds stress anisotropy $b_{11},(e)$ turbulence kinetic energy $k,(f)$ dissipation $\epsilon$.

growth rate. Thus the increase in $b_{11}$ at high Mach numbers limits the growth rate reduction through the slow pressure-strain term.

As has been remarked, our simulations are not fully self-similar and this partially explains the scatter of the DNS data visible in figures 8 and 9 (compare figure 5). Another reason could be the anisotropy of the dissipation in the simulations, but not included in the model since it is expected to vanish at very high Reynolds numbers. Although not very large, the dissipation anisotropy is most significant at $M=1.2$ and according to equation (26) it has a positive contribution to the growth rate. Hence the DNS growth rate in figure 8 for $M=1.2$ is indeed expected to be slightly larger than the model prediction.

The model is well-conditioned, i.e. not very sensitive to variations in the model constants. Additional calculations of the growth rate have been performed in which 
each of the model constants was changed separately. The relative variations of the growth rate were approximately equal to or less than the relative changes of the model constants.

\section{Discussion}

One important observation in this paper has been that dilatation dissipation and pressure dilatation are not large in the compressible mixing layer, even when eddy shocklets are present. Turbulence models constructed using dilatation dissipation or pressure dilatation are not necessarily invalidated by this, but their claim to be based on the correct physics of the flow would now appear to be false. Some of the initial support for the dilatation dissipation concept came from simulations of isotropic or fully homogeneous compressible turbulence. Eddy shocklets were observed in isotropic and homogeneous shear flow (Lee, Lele \& Moin 1991; Blaisdell et al. 1991) and the magnitude of the dilatational contribution to dissipation was computed and compared with models for dilatational dissipation, for example that of Sarkar et al. (1991). Blaisdell et al. found the model by Sarkar et al. to be accurate for turbulent Mach numbers below 0.3. For larger turbulent Mach numbers the dilatation dissipation was found to be constant at a level of no more than $10 \%$ of the total dissipation. Also Lee et al. (1991) found only 5\% dilatation dissipation at a turbulent Mach number of 0.5 , whereas Sarkar's model would predict $25 \%$. Thus, even in isotropic and homogeneous shear flow, the dilatational terms cannot be regarded as essential in causing reduced growth rates. This is confirmed by recent work on homogeneous shear flow (Sarkar 1995), in which compressibility was found to affect other terms (e.g. production) more than the dissipation. Another recent confirmation is the work by Simone \& Cambon (1995), which shows that the effect of compressibility is reflected in pressure-strain correlations and related to the anisotropy of the Reynolds stress tensor, rather than in explicit dilatation terms such as pressure-dilatation and dilatation dissipation. The subject of the latter paper is also homogeneous shear flow, studied by means of DNS and rapid distortion theory (Durbin \& Zeman 1992; Cambon et al. 1993; Jacquin, Cambon \& Blin 1993). Furthermore, a different form of splitting the dissipation into solenoidal and dilatational parts has recently been proposed (Huang 1995). This decomposition would predict even smaller dilatational effects.

The mixing layer is very strongly affected by compressibility. Using the model from this paper, this can be explained by the large pressure fluctuations of the typical eddies in the flow. Similarly large pressure fluctuations and hence Mach number sensitivity would be found in jet and wake flows. Other flows, such as the turbulent boundary layer on a wall, have comparatively much weaker pressure fluctuations and the effects of compressibility do not appear until much higher Mach numbers.

In this paper we did not intend to develop a turbulent field model, applicable to a general turbulent flow. We have analysed and modelled the integrated Reynolds stress equations in order to determine the growth rate for the mixing layer. The model for the integrated pressure-strain presented in this paper is (via $p^{*}$ ) a function of the convective Mach number $M$. Although this Mach number is not a field quantity, it is closely related to a field quantity, the local gradient Mach number $M_{g}$ (Sarkar 1995). Whether the gradient Mach number could play a role in a turbulent field model needs further exploration.

The agreement between the actual level of the pressure fluctuations (figure 6) and the model predictions (figure 7) is reasonable. The main difference is $p^{+}$at high Mach 


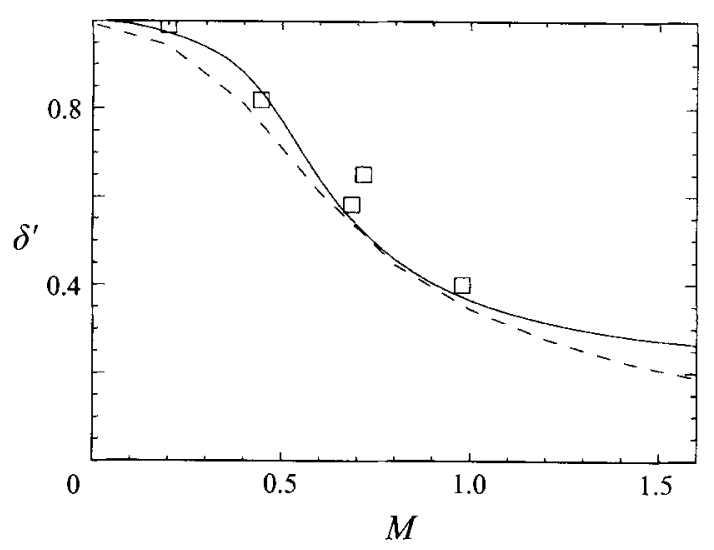

FigURE 10. Normalized growth rate against Mach number comparing the current model (solid line) with the linear stability result (dashed line) and experimental data from Goebel \& Dutton (1991) (symbols).

numbers which is larger than the model predicts. This could be due to different types of eddies contributing to the pressure field. There are eddies that contribute to the rapid pressure-strain (the part that depends on mean velocity gradients and this part is modelled in \$4.2) and eddies that contribute to the slow part. For the latter types of vortices the pressures may be higher than those obtained with the isentropic model. Furthermore, shocks will also give rise to higher pressure maxima than predicted with isentropic assumptions, since the entropy of the fluid increases when passing through a shock. The high pressure then comes from a supersonic eddy which generates a shock wave. Supersonic eddies may be present in the flow, but according to Breidenthal (1990) do not have time to rotate and contribute to entrainment and growth rate. If the parameter $p^{*}(M)$ is corrected for higher pressures at higher Mach numbers, the predicted growth rate will be somewhat larger. Reasonable agreement with experimental and simulation data is nevertheless still obtained, if $p_{\max }=p_{\infty}+\frac{1}{2} \rho_{\infty}\left(\frac{1}{2} \Delta U\right)^{2}$ is substituted in equation (31), assuming that $p^{+}$does not exceed its incompressible value.

In the introduction it was remarked that the reduction in growth rates with Mach number for the most unstable waves from linear stability theory matches almost exactly the reduction in shear layer growth rate of the fully turbulent flow. In figure 10 we compare the growth rate reduction from temporal stability theory with the result from the turbulence model of $\$ 4.3$ and experimental data from Goebel \& Dutton (1991). The latter is chosen because the density ratio across the shear layer is always less than 2:1, so that these data are comparatively insensitive to the model for incompressible growth rate used for normalization. As can be seen there is a good correlation between the two curves and the experiments.

The growth rate $\omega_{i}$ in the linear regime and the growth rate of the momentum thickness $\delta^{\prime}$ in the turbulent regime are not defined in the same way. In the linear regime we have an exponential growth of the amplitude of the disturbance. Except from the effect of molecular dissipation, the width of the profiles does not change and the momentum thickness does not grow. In the nonlinear turbulent regime however, there is a constant growth of the thickness of the profiles (and the momentum thickness), whereas the amplitude of the perturbations has evolved towards a saturated state. Hence, it is difficult to explain the reduction in $\delta^{\prime}$ directly 
by the reduction in $\omega_{i}$. However, in the following it does appear that the terms in the integrated Reynolds stress transport equations that are reduced in the turbulent stage are also reduced in the linear stage. In particular the pressure fluctuations at increased Mach number also show a reduction in the linear stage. To establish this we apply the mathematical averaging procedure of $\$ 3$ (average over the streamwise and spanwise directions) to the fluctuations in the linear stability regime.

In temporal linear stability theory of the mixing layer the fluctuating variables have the following form:

$$
\phi(x, y, z, t)=A\left(\hat{\phi}^{r}\left(x_{2}\right)+\mathrm{i} \hat{\phi}^{i}\left(x_{2}\right)\right) \mathrm{e}^{\left(\omega_{i} t+\mathrm{i}\left(\alpha x_{1}+\beta x_{3}\right)\right.},
$$

where $A$ is a small amplitude, $\omega_{i}$ is the temporal growth rate, $\alpha$ and $\beta$ are the streamwise and spanwise wavenumbers, $i$ is the complex number and $\hat{\phi}^{r}$ and $\hat{\phi}^{i}$ are the real and imaginary part of the eigenfunction $\hat{\phi}$. The integrated diagonal Reynolds stress equations reduce to

$$
\begin{aligned}
& \omega_{i}\left\|\hat{u}_{1}\right\|^{2}=\hat{P}_{11}+\hat{\Pi}_{11}, \\
& \omega_{i}\left\|\hat{u}_{2}\right\|^{2}=\hat{\Pi}_{22}, \\
& \omega_{i}\left\|\hat{u}_{3}\right\|^{2}=\hat{\Pi}_{33},
\end{aligned}
$$

where $\|\hat{\phi}\|^{2}=\int|\hat{\phi}|^{2} \mathrm{~d} x_{2}$ and the terms on the right-hand side reflect production and pressure-strain:

$$
\begin{aligned}
& \hat{P}_{11}=-\int\left(\hat{u}_{1}^{r} \hat{u}_{2}^{r}+\hat{u}_{1}^{i} \hat{u}_{2}^{i}\right) \frac{\mathrm{d} \bar{u}_{1}}{\mathrm{~d} x_{2}} \mathrm{~d} x_{2}, \\
& \hat{\Pi}_{11}=\alpha \int\left(\hat{p}^{i} \hat{u}_{1}^{r}-\hat{p}^{r} \hat{u}_{1}^{i}\right) \mathrm{d} x_{2}, \\
& \hat{\Pi}_{22}=\int\left(\hat{p}^{r} \frac{d \hat{u}_{2}^{r}}{d x_{2}}+\hat{p}^{i} \frac{d \hat{u}_{2}^{i}}{d x_{2}}\right) \mathrm{d} x_{2}, \\
& \hat{\Pi}_{33}=\beta \int\left(\hat{p}^{i} \hat{u}_{3}^{r}-\hat{p}^{r} \hat{u}_{3}^{i}\right) \mathrm{d} x_{2} .
\end{aligned}
$$

In the derivation we have assumed $\bar{\rho}=1$ following Blumen (1970). The pressure eigenfunction satisfies the following relation (Blumen 1970):

$$
\omega_{i} M^{2}\|\hat{p}\|^{2}=-\hat{\Pi}_{k k} \text {. }
$$

We observe that positive growth rate implies $\hat{\Pi}_{22}>0, \hat{\Pi}_{33}>0, \hat{\Pi}_{k k}<0$ and, consequently, $\hat{\Pi}_{11}<0$. Furthermore, negative $\hat{\Pi}_{11}$ implies positive $\hat{P}_{11}$. Hence, the production and pressure-strain terms in the linear regime have the same sign as in the turbulent regime.

We have solved the linear stability problem for several Mach numbers from 0 up to 1.6 and obtained the most unstable mode normalized with $\left\|\hat{u}_{1}\right\|=1$ for each Mach number. The pressure fluctuation $\|\hat{p}\|$ was observed to drop rapidly with Mach number. This is expected from equation (54), since $\left|\hat{\Pi}_{k k}\right|<\left|\hat{\Pi}_{11}\right| \sim\|\hat{p}\|\left\|\hat{u}_{1}\right\|$. To maximize the growth rate at high Mach number the pressure fluctuations should become smaller. Up to $M=0.6$ the production and pressure-strain were observed to decrease in exact proportion to the growth rate $\omega_{i}$. Above $M=0.6$ we have a change from two-dimensional to oblique disturbances in the stability theory. In this regime the pressure-strain was observed to drop more rapidly than production and both dropped more rapidly than the linear growth rate. 
Hence, although the linear $\omega_{i}$ and turbulent $\delta^{\prime}$ are different quantities, the reduction at increased Mach number is in both cases connected to reduced pressure fluctuations, reduced production and pressure-strain and increased anisotropy of the streamwise fluctuations.

\section{Conclusions}

Detailed analysis has been made of direct simulation databases of compressible mixing layers with convective Mach number $M$ ranging from 0.2 to 1.2. The simulations showed a reduction in growth rate matching the reduction found in previous experimental work. Instantaneous realizations of the flow at $M=1.2$ revealed the presence of eddy shocklets. However, statistics for dilatation dissipation and pressuredilatation, which might be expected to become important because of the shocklets, showed that these were much smaller than the total dissipation. Therefore the eddy shocklets, and the dilatational terms in the averaged equations, were not found to be significant for understanding the reduced growth rate.

Analysis of integrated statistics, incorporating a relation between the integrated production and the shear layer growth rate, showed that the reduction in growth rate was due to a reduction in the pressure-strain term. A model was then developed based on reduced pressure fluctuations. Values for pressure were deduced from a deterministic model for typical compressible eddies. Simple anisotropy considerations closed the model which was then demonstrated to predict the variation of integrated Reynolds stresses, pressure-strain terms, and dissipation, in good agreement with the direct simulation data.

A. W. Vreman would like to thank Stichting Nationale Computerfaciliteiten (National Computing Facilities Foundation, NCF) for computer time and the Nederlandse Organisatie van Wetenschappelijk Onderzoek (Netherlands Organization for Scientific Research) for financial support of this research project. Support and computer time for K. H. Luo came from the UK Engineering and Physical Sciences Research Council (EPSRC), grant GR/H 40518.

\section{REFERENCES}

BiRCH, S. F. \& EGgers, J. M. 1973 A critical review of the experimental data for developed free turbulent shear layers. NASA SP-321, pp. 11-40.

Blaisdell, G. A., Mansour, N. N. \& Reynolds, W. C. 1991 Numerical simulation of compressible homogeneous turbulence. Rep. TF-50. Department of Mechanical Engineering, Stanford University.

BLumen, W. 1970 Shear layer instability of an inviscid compressible fluid. J. Fluid Mech. 40, 769-781.

Bogdanoff, D. W. 1983 Compressibility effects in turbulent shear layers. AIAA J. 21, 926-927.

Bradshaw, P. 1977 Compressible turbulent shear layers. Ann. Rev. Fluid Mech. 9, 33-54.

BREIDENTHAL, R. 1990 The sonic eddy - a model for compressible turbulence. AIAA paper 90-0495.

BrowaNd, F. K. \& LATIGO, B. O. 1979 Growth of the two-dimensional mixing layer from a turbulent and nonturbulent boundary layer. Phys. Fluids 22, 1011-1019.

Brown, G. L. \& Roshko, A. 1974 On density effects and large structure in turbulent mixing layers. J. Fluid Mech. 64, 775-816.

Burgers, J. M. 1948 A mathematical model illustrating the theory of turbulence. Adv. Appl. Mech. $1,171-196$.

Cambon, C., Coleman, G. N. \& Mansour, N. N. 1993 Rapid distortion analysis and direct simulation of compressible homogeneous turbulence at finite Mach number. J. Fluid Mech. 257, 641-665. 
Clemens, N. T. \& Mungal, M. G. 1992 Two- and three-dimensional effects in the supersonic mixing layer. $A I A A J . \mathbf{3 0}, 973-981$.

Clemens, N. T. \& Mungal, M. G. 1995 Large-scale structure and entrainment in the supersonic mixing layer. J. Fluid Mech. 284, 171-216.

Durbin, P. A. \& Zeman, O. 1992 Rapid distortion theory for homogeneous compressed turbulence with application to modelling. J. Fluid Mech. 242, 349-370.

El Baz, A. M. \& Launder, B. E. 1993 Second-moment modelling of compressible mixing layers. Engineering Turbulence Modelling and Experiments 2 (ed. W. Rodi \& F. Martelli). Elsevier.

Elliot, G. S. \& Samimy, M. 1990 Compressibility effects in free shear layers. Phys. Fluids A 2, $1231-1240$.

Gibson, M. M. \& Launder, B. E. 1978 Ground effect in the atmospheric boundary layer. J. Fluid Mech. 86, 491-511.

Goebel, S. G. \& Dutton, J. C. 1991 Experimental study of compressible turbulent mixing layers. AIAA J. 29. 538-546.

HUANG, P. G. 1995 Relations between viscous diffusion and dissipation of turbulent kinetic energy. Proc. Turbulent Shear Flows 10, The Pennsylvania State University, pp. P2.79-84.

Jacquin, L., Cambon, C. \& Blin, E. 1993 Turbulence amplification by a shock wave and rapid distortion theory. Phys. Fluids A 5, 2539-2550.

Lee, S., Lele, S. K. \& Morn, P. 1991 Eddy shocklets in decaying compressible turbulence. Phys. Fluids A 3, 657-664.

LeLE, S. K. 1994 Compressibility effects on turbulence. Ann. Rev. Fluid Mech. 26, 211-254.

LU, G. \& LELE, S. K. 1994 On the density ratio effect on the growth rate of a compressible mixing layer. Phys. Fluids 6, 1073-1075.

Luo, K. H. \& Sandham, N. D. 1994 On the formation of small scales in a compressible mixing layer. In Direct and Large-Eddy Simulation I, pp. 335-346. Kluwer.

Luo, K. H. \& Sandham, N. D. 1995 Reynolds number effects on transitional compressible free shear flows: direct numerical simulation. In Proc. First Asian Computational Fluid Dynamics Conference, Hong Kong, January 16-19, pp. 403-408.

Moser, R. D. \& Rogers, M. 1993 The three-dimensional evolution of a plane mixing layer: pairing and transition to turbulence. J. Fluid Mech. 247, 275-320.

Papamoschou, D. 1995 Evidence of shocklets in a counterflow supersonic shear layer. Phys. Fluids 7, 233-235.

Papamoschou, D. \& Lele, S. K. 1992 Vortex-induced disturbance field in a compressible shear layer. CTR Proc. of the Summer Program 1992, pp. 259-276.

Papamoschou, D. \& Roshko, A. 1988 The compressible turbulent mixing layer: an experimental study. J. Fluid Mech. 197, 453-477.

Ragab, S. A. \& ShEen, S. 1992 The nonlinear development of supersonic instability waves in a mixing layer. Phys. Fluids A 4, 553-566.

RistoRCELLI, J. R. 1995 A pseudo-sound constitutive relationship for the dilatational covariances in compressible turbulence: an analytical theory. ICASE Rep. 95-22. NASA Langley Research Center, Hampton.

Rogers, M. M. \& Moser, R. D. 1994 Direct simulation of a self-similar turbulent mixing layer. Phys. Fluids 6, 903-924.

Sandham, N. D. \& Reynolds, W. C. 1989 A numerical investigation of the compressible mixing layer. Rep. TF-45. Department of Mechanical Engineering, Stanford University.

Sandham, N. D. \& Reynolds, W. C. 1990 Compressible mixing layer: Linear theory and direct simulation. AIAA J. 28, 618-624.

Sandham, N. D. \& Reynolds, W. C. 1991 Three-dimensional simulations of large eddies in the compressible mixing layer. J. Fluid Mech. 224, 133-158.

Sarkar, S. 1995 The stabilizing effect of compressibility in turbulent shear flow. J. Fluid Mech. 282, 163-186.

Sarkar, S., Erlebacher, G., Hussainl, M. Y. \& Kreiss, H. O. 1991 The analysis and modelling of dilatational terms in compressible turbulence. J. Fluid Mech. 227, 473-493.

Simone, A. \& CAMBON, C. 1995 Rapid distortion and direct simulation approach to compressibility in turbulent shear flow. Proc. Turbulent Shear Flows 10, The Pennsylvania State University.

Speziale, C. G., Sarkar, S. \& Gatski, T. B. 1991 Modelling the pressure-strain correlation of turbulence: an invariant dynamical systems approach. J. Fluid Mech. 227, 245-272. 
Tennekes, H. \& Lumley, J. L. 1972 A First Course in Turbulence. The MIT Press.

VREMAN, B. 1995 Direct and large-eddy simulation of the compressible turbulent mixing layer. $\mathrm{PhD}$ Dissertation, University of Twente.

Vreman, B., Geurts, B. \& Kuerten, H. 1995a Subgrid-modelling in LES of compressible flow. Appl. Sci. Res. 54, 191-203.

VReman, B., Kuerten, H. \& GeURTs, B. $1995 b$ Shocks in direct numerical simulation of the confined three-dimensional mixing layer. Phys. Fluids 7, 2105-2107.

ZEMAN, O. 1990 Dilatation dissipation: The concept and application in modeling compressible mixing layers. Phys. Fluids A 2, 178-188.

ZEMAN, O. 1991 On the decay of compressible isotropic turbulence. Phys. Fluids A 3, 951-955. 